\title{
Caracterización del binomio adulto mayor-familiar de convivencia
}

\author{
Characterization of binomial older \\ adult-relative of coexistence
}

\begin{abstract}
Dra. Rosa Ma. Aguilar Hernández*, MSP. Aurora Félix Alemán*, MES Ma. de la Luz Martínez Aguilar**, MCE Ma. Del Carmen Vega Alanis**, MES Ma. Magdalena Quevedo Díaz, ME Gustavo Gutiérrez Sánchez* *Profesores de tiempo completo, integrantes del Cuerpo Académico Salud y Comportamiento Humano de la Unidad Académica Multidisciplinaria Matamoros-UAT (UAMM-UAT), **Profesores de tiempo completo, colaboradores del cuerpo Académico Salud y Comportamiento Humano de la UAMM-UAT
\end{abstract}

\section{Resumen}

Introducción: El incremento de población adulto mayor exige responsabilidad sanitaria y familiar para que el aumento en años de vida sea de vida saludable.

Objetivo: Identificar las características del adulto mayor y del familiar de convivencia como base para las acciones.

Metodología: Estudio descriptivo, transversal con 96 binomios adulto mayor-familiar de convivencia. Cuestionario de datos biográficos y de salud para el adulto mayor y datos socios demográficos para el familiar, aplicados mediante entrevista en los hogares. Se utilizó el Programa Estadístico SPSS Versión 17. Resultados: Relación significativa en edad y sexo entre adultos mayores y familiares de convivencia. Media de edad 70 y 45 ; sexo, mujeres $59.4 \%$ y $67.7 \%$; casados o en unión libre $56.3 \%$ y $72.9 \%$, respectivamente. Familiar de convivencia hijo/a (54 \%) y esposo/a (32\%) mayoría mujeres con bajo nivel de escolaridad (65\%) y ocupación labores del hogar (64\%).

La convivencia es favorable para la salud porque hay un mayor control de las conductas de riesgo. Relevancia de las características del binomio adulto mayor-familiar de convivencia para las acciones de promoción y prevención en los hogares, orientadas a fortalecer la relación y desarrollar habilidad en la atención al adulto mayor.

Palabras clave: Adulto mayor, familia, convivencia

\section{Abstract}

Abstract Nurses have sensitivity, knowledge and experience to attend the binomial older adult-relative of coexistence in family context. The purpose of the study was to identify characteristics of the older adult and the relative of coexistence as base to plan actions. The study was descriptive, crosssectional with 96 binomials adult major-relative of greater coexistence. Biographical data and health questionnaire for the older adult and demographic data for the relative applied by means of interview in the homes. We used the statistical program SPSS version 17. Age and sex had significant relation between older adults and relatives of coexistence. Age average, 70 and 45; woman, 59. 4\% and 67.7\%; ma- rried, $56.3 \%$ and 72.9 , respectively. Relative of coexistence was child (54\%) and wife or husband (32\%), majority were woman with low level of education (65\%) and occupation of the household work (64\%).

The coexistence is favorable for the health because there is a greater control of the risk conducts. Relevance of the characteristics of the binomial older adult-relative of coexistence for promotion and prevention actions in the homes, oriented to fortify the relation and to develop ability in the attention to the older adult.

Keywords: Older adult, family, coexistence 


\section{INTRODUCCIÓN}

El envejecimiento poblacional es un fenómeno mundial que representa un desafío para los gobiernos y los sistemas de salud, en particular de los países en desarroIlo, por el incremento acelerado de las personas de 60 años y más, así como por la falta de preparación para enfrentar el fenómeno. En este grupo de edad se encuentran más de 600 millones de personas en el mundo, cifra que, de acuerdo con las predicciones, se duplicará en los próximos 15 años y alcanzará los dos billones para el año $2050^{1}$.

La esperanza de vida reportada para los mexicanos es de 74.8 años (72.4 hombres y 77.2 mujeres) ${ }^{4}$. En casi la cuarta parte de los hogares mexicanos hay uno o más adultos mayores ${ }^{5}$. El incremento de los años de vida y del grupo de población de adultos mayores conlleva tomar responsabilidad política, social, sanitaria y familiar para enfrentar el problema, de manera que el aumento en años de vida sea también de vida saludable ${ }^{3}$.

El envejecimiento es un proceso natural en la vida que conlleva deterioro anatomofisiológico paulatino y progresivo, que se manifiesta por la disminución de la vitalidad, aumento de la fragilidad y vulnerabilidad del adulto mayor a factores ambientales y psicosociales ${ }^{7,8}$. Esto hace que sea común vincular al adulto mayor con la enfermedad, aunque no siempre están ligados.

El adulto mayor sano es definido por Pietro de Nicola ${ }^{9}$ como una "persona con alteraciones morfológicas y funcionales en el límite entre lo normal y lo patológico, en equilibrio inestable y con adaptación a la capacidad funcional y a las posibilidades reales de rendimiento". Los términos clave son conocimiento sobre los cambios y adaptación a ellos; con el ingrediente insubstituible que es el apoyo de la familia.

La familia es el referente social y grupo de pertenencia más importante de las personas ${ }^{10}$; concepto de relevancia para el adulto mayor; ya que por el decline en sus funciones necesita del apoyo para realizar sus actividades ${ }^{11}$. De ahí que la salud del adulto mayor se sustente en un ambiente familiar de apoyo, comprensión y comunicación.'

Un ambiente familiar de afecto, cariño y atención es favorable para la salud; por el contrario, el contexto familiar de desapego y desinterés conduce a sentimientos de abandono y surge la depresión que es campo propicio para las enfermedades; que cuando se establecen como crónicas modifican la vida cotidiana no sólo del adulto mayor sino también de la familia ${ }^{12}$.
El recurso más importante que los adultos mayores valoran y añoran de su familia es el tiempo de convivencia; sin embargo, esto es lo que las familias de la agitada vida moderna menos dispensan. Los adultos mayores y los miembros de las generaciones más jóvenes se mueven a ritmos diferentes; los primeros toman la vida con mayor calma; los segundos la viven de prisa y no se dan tiempo para fomentar la convivencia.

La familia debe conocer los beneficios de convivir con los adultos mayores, de apoyarlos en sus necesidades, de brindarles afecto y comprensión para mantener su autoestima, fomentar el auto cuidado y con ello la salud. En la familia se gesta la salud o la enfermedad y lo que el adulto mayor manifiesta en forma abierta o encubierta es una evidencia de lo que su familia le brinda.

El interés de los profesionales de la salud por estudiar a los adultos mayores, en particular de enfermería, se muestra en el número de reportes de investigación sobre este grupo de población. En la revisión de literatura se encontraron estudios que abordan la dependencia del adulto mayor relacionado con otras variables ${ }^{13,14,15}$. Otros tratan aspectos como las características del adulto mayor ${ }^{16}$, su estado mental ${ }^{17}$, percepción de la calidad de atención de enfermería ${ }^{18}$ y la satisfacción con dicha atención en relación con variables socio demográficas de los adultos mayores ${ }^{19}$. Sin embargo, no se han encontrado estudios que caractericen al binomio adulto mayor-familiar de convivencia.

En este estudio se utiliza el término familiar de convivencia porque, a diferencia de la mayoría de los estudios con adultos mayores, el interés no se centró en la dependencia o en alguna patología sino en la convivencia; considerada un indicador de la calidad de atención que reciben los adultos mayores de la familia.

El concepto familiar de convivencia tiene una connotación positiva, porque se relaciona con un adulto mayor funcional. A diferencia del cuidador familiar que asume la responsabilidad de cuidar al adulto mayor dependiente, para lo cual generalmente no se encuentra preparado.

Asumir esta responsabilidad no resulta libre de tensiones y puede ser repentina, sobre todo cuando la relación previa del adulto mayor y su familiar no fue favorable ${ }^{20} \cdot \mathrm{La}$ relación que tiene el familiar de convivencia, con el adulto mayor que se vuelve dependiente, aumenta la probabilidad de que se convierta en su cuidador/a ${ }^{21}$.

Enfermería es el grupo profesional con sensibilidad, conocimiento y competencia para el cuidado del adulto mayor en su entorno familiar; por lo que es capaz de sen- 
sibilizar y preparar al familiar de convivencia en la atención de las necesidades del adulto mayor para mantener la independencia.

El punto de partida es conocer las características de los adultos mayores y del familiar de convivencia en sus hogares, para, planear y poner en práctica acciones preventivas y de promoción a la salud con el binomio adulto mayor-familiar de convivencia. Por esta razón, se hace necesaria la generación de conocimientos que impacten en la formación de profesionales de enfermería; de manera que aborden los problemas de la práctica, en donde los adultos mayores por su incremento poblacional serán los que requerirán servicios preventivos de calidad en el contexto familiar. El eje de las acciones lo constituye la educación para la salud, con base en el conocimiento sobre los cambios y la necesidad de adaptación del adulto mayor y de la familia en esta etapa de vida. De esta manera será posible lograr una vida saludable a pesar de las limitaciones propias del envejecimiento, para evitar o retardar la hospitalización y el incremento de los costes sanitarios y de recursos familiares.

El objetivo del estudio fue identificar las características del binomio adulto mayor-familiar de convivencia; conocimiento que será la base para la planeación del cuidado de enfermería al adulto mayor en su contexto familiar.

\section{METODOLOGÍA}

El estudio fue descriptivo, transversal con muestreo de conveniencia de 96 binomios adulto mayor-familiar de convivencia. El estudio forma parte de un proyecto mayor, con intervención educativa en familias con adultos mayores. Se incluyeron adultos de 60 años y mayores, de ambos sexos, miembros de familias de dos o más integrantes, residentes del área de influencia del Centro Comunitario Las Culturas, con seis meses o más de residir en el grupo familiar actual. Los criterios para seleccionar al familiar de convivencia fueron edad de 18 años o más, residir en el mismo domicilio del adulto mayor, ambos con disposición para participar en la entrevista. Para este estudio solo se tomaron los datos socio demográficos del adulto mayor y del familiar, obtenidos mediante cuestionario de respuesta abierta y breve. Los reactivos fueron, además de los datos de identificación, cuestiones acerca de parentesco, religión, percepción de salud, eventos significativos y datos médicos relevantes del adulto mayor. Para el familiar las preguntas fueron solo sobre datos de identificación y parentesco con el adulto mayor. Estos instrumentos, muy sencillos en su construcción, fueron probados, acerca de la claridad de las preguntas y la pertinencia de las respuestas al objetivo del estudio, fueron avalados mediante la prueba piloto de los cuestionarios, aplicados a 30 binomios con características similares a los de la muestra. Los cuestionarios fueron aplicados mediante entrevista en los hogares.

El trabajo de campo fue coordinado por la líder del estudio y realizado por estudiantes del último semestre de Licenciatura en Enfermería. Una vez confirmados los criterios de inclusión y obtenido el consentimiento informado se aplicaron los instrumentos. Las consideraciones éticas del estudio fueron cubiertas en apego a lo establecido en los artículos 21 y 22 del Reglamento de la Ley General de Salud en materia de investigación para la salud de la Secretaría de Salud ${ }^{22}$

Para el procesamiento de la información se utilizó el Programa Estadístico SPSS Versión 17. Para analizar la relación entre variables de tipo nominal se utilizó la prueba exacta de Fisher y la relación de las variables continuas se estudió a través del coeficiente de correlación lineal de Pearson.

\section{RESULTADOS}

\section{Edad del adulto mayor y familiar de convivencia}

Los adultos mayores estudiados comprenden edades de 60 a 95 años, con una media de 70 , mientras que los familiares abarcan un rango más amplio que va desde los 18 a los 83 años, con una media de 45. Existe una relación significativa entre la edad del adulto mayor y del familiar de convivencia $(r=0.268, p=0.0084)$ (Figura. 1).

\section{Figura 1. Relación entre la edad del adulto mayor y familiar de convivencia}

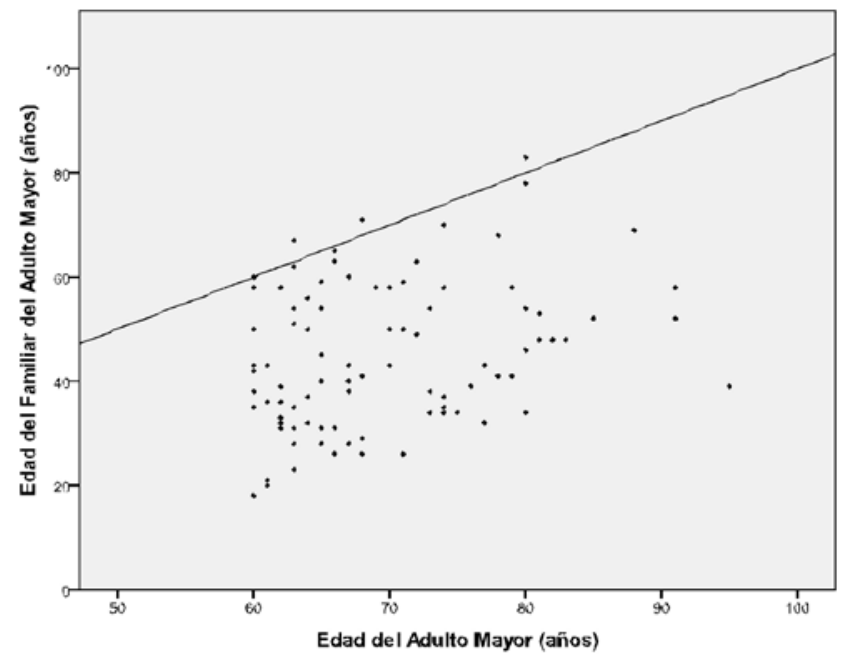


En la mayoría de los casos el familiar de convivencia es de menor edad que el adulto mayor; sólo en cinco casos el familiar de convivencia tiene una edad superior o igual a la del adulto mayor.

\section{Sexo del adulto mayor y familiar de convivencia}

Las mujeres son mayoría tanto en el grupo de adultos mayores (59.4\%) como en el de familiar de convivencia (67.7\%). Con una relación estadísticamente significativa entre el sexo del adulto mayor y del familiar de convivencia $(p=0.0001)$ (Tabla 1). En la mayoría de los casos el familiar de convivencia del sexo femenino es quien se encarga del adulto mayor en una relación de 2.1 a 1 . Sin embargo, esta relación es menor cuando se trata de un adulto mayor del sexo femenino, 1.1 a 1; seguramente debido a que es el esposo conviviente de la mujer, si no se incluyen estos casos, la relación es de 1.5 a 1.

\section{Estado civil del adulto mayor y familiar de convivencia}

La mayoría de adultos mayores (43\%) y familiares de convivencia (64\%) es casado/a. Sin embargo, si se incluye a los que viven en unión libre, la vida en pareja es mayor tanto para los adultos mayores (56.3\%) como para los familiares de convivencia (72.9\%). Un poco menos de un tercio de los adultos mayores es viudo/a (31.3\%).

\section{Ocupación del adulto mayor y familiar de convivencia}

La mayoría de los adultos mayores tiene ocupación actual (99\%), más de la mitad (64\%) se ocupa en labores del hogar, en particular mujeres. En cuanto al familiar de convivencia, casi la mitad (46\%) tiene como ocupación las labores del hogar, en su mayoría mujeres.

\section{Religión del adulto mayor}

La mayoría (68\%) son católicas con una amplia diferencia con respecto a los cristianos que es la segunda categoría (16\%); evangélicos, testigos de Jehová y otras religiones representan menos del 10\% cada una.

\section{Percepción de salud del adulto mayor}

La mayoría de los adultos mayores (91\%) percibe su salud entre regular (42\%) y buena (49\%). La mayoría de los adultos mayores $(90 \%)$ refiere más de un problema de salud relevante; en más de la mitad se trata de una enfermedad crónico degenerativa. Más de dos tercios de los adultos mayores (76\%) refiere un evento de vida significativo entre los que se encuentran muerte, enfermedad y cirugía.

\section{Escolaridad del familiar de convivencia}

Los familiares tienen como promedio 6 años de estudio (igual a la mediana) con una desviación estándar de 3.99 años. Esto está de acuerdo con el hecho que más de la mitad de los familiares convivientes (51\%) sólo tiene estudios primarios. Son pocos los casos con estudios superiores, entre preparatoria o equivalente y universidad (14\%), porcentaje similar al de quienes tienen una escolaridad nula.

\section{Convivencia}

La mitad de los adultos mayores (51.1\%) vive con tres o más personas. El parentesco de los familiares de convivencia con el adulto mayor es de: hijo/a (54\%), cónyuge (32\%), hijos políticos, nietos y otros familiares son minoría (13\%). Vive con su cónyuge (48\%); de éstos, la mayoría vive también con un hijo, hijo político o nieto. En estos casos, a pesar de que el adulto mayor vive con el cónyuge, el familiar de convivencia es hijo/a (13\%). Un porcentaje similar (46\%) vive solo con un hijo, hijo político o nieto.

Tabla 1. Distribución por sexo del adulto mayor y familiar de convivencia. La línea de referencia corresponde a la relación 1:1

\begin{tabular}{|c|c|c|c|c|c|c|c|}
\hline & & & \multicolumn{4}{|c|}{ Familiar de convivencia } & \multirow{3}{*}{ Total } \\
\hline & & & \multicolumn{2}{|c|}{ Masculino } & \multicolumn{2}{|c|}{ Femenino } & \\
\hline & & & $\mathbf{n}$ & $\%$ & $\mathbf{n}$ & $\%$ & \\
\hline \multirow{4}{*}{ Adulto mayor } & Masculino & $n$ & 4 & 10 & 35 & 90 & 39 \\
\hline & & $\%$ & 13 & & 54 & & 41 \\
\hline & Femenino & $\mathrm{n}$ & 27 & 47 & 30 & 53 & 57 \\
\hline & & $\%$ & 87 & & 46 & & 59 \\
\hline \multirow{2}{*}{\multicolumn{2}{|c|}{ Total }} & $n$ & 31 & 32 & 65 & 68 & 96 \\
\hline & & $\%$ & 32 & & 68 & & \\
\hline
\end{tabular}


Más de la mitad de los adultos mayores (55.2\%) refiere tener, además de los familiares que viven en la misma casa, otra persona significativa; de los cuales, son hijos (26\%), nietos (4.2\%) y otros (25\%). De estos adultos mayores, casi dos tercios vive con una sola persona (65\%). La mayoría de los que viven con dos o más familiares $(80 \%)$, no refiere tener otra persona significativa.

\section{DISCUSIÓN}

Predominan las mujeres en el grupo de adultos mayores, similar a lo encontrado por Barrrios y Cols. ${ }^{16}$, pero diferente a lo reportado por Filgueredo y cols. ${ }^{17}$ quienes encontraron poco más de la mitad de adultos mayores del sexo masculino.

Destaca el hecho que más de un tercio de los familiares de convivencia son también adultos mayores y que casi la cuarta parte se encuentra en la década previa, esto puede significar que el binomio adulto mayor-familiar de convivencia se comprende y participa de la experiencia de envejecer; aunado al hecho de que más de la mitad de los adultos mayores del estudio vive en pareja. Estos son aspectos favorables para la salud, ya que de acuerdo con La Parra ${ }^{23}$, la vida en pareja favorece el cuidado mediante el control de las conductas de riesgo para la salud.

La mayoría de los adultos mayores del estudio que viven con dos o más familiares no tienen otra persona significativa en su vida. Estos resultados sugieren que un mayor número de personas en la familia favorece el bienestar de los adultos mayores ya que de acuerdo con Salgado y Wong ${ }^{(24)}$ la vejez se vive de forma diferente si se está solo o acompañado por la esposa o por los hijos.

La mayoría de los adultos mayores percibe buen estado de salud, mientras que la mitad de ellos presenta enfermedad crónica degenerativa, este dato se refleja en que se mantienen activos, ya que la mayoría reporta ocupación actual, principalmente en labores del hogar. La Organización Mundial de la Salud ${ }^{1}$ promueve el envejecimiento activo, para mantener la funcionalidad y el bienestar.

Es muy probable que los familiares de convivencia de este estudio se conviertan en cuidadores familiares, porque tienen algunos rasgos que los caracterizan: la mayoría son mujeres, con bajo nivel o nula escolaridad y se ocupan de las labores del hogar en su mayoría, estas características han sido identificadas en estudios realizados por el Ministerio de Trabajo y Asuntos Sociales, de España ${ }^{25}$. De acuerdo con Bover y Gastaldo ${ }^{20}$, las mujeres asumen de manera natural el cuidado de los adultos mayores.

\section{Implicaciones para la práctica}

Los adultos mayores son frágiles y, por consecuencia, susceptibles de daños, por lo que deben ser atendidos por los familiares de convivencia. El deterioro progresivo por el envejecimiento es inevitable, por lo que las familias necesitan estar preparadas y sensibilizadas para la convivencia y atención de sus adultos mayores. Los profesionales de enfermería cuyo objeto de atención es el cuidado a la salud, en particular de los grupos vulnerables, tienen el compromiso de promover la salud y prevenir la enfermedad del binomio adulto mayor-familiar de convivencia. Las enfermeras, con base en el conocimiento sobre el fenómeno del envejecimiento y las repercusiones en la familia, pueden anticiparse al problema que representa el binomio adulto mayor dependiente-cuidador familiar mediante el fomento de la relación adulto mayor-familiar de convivencia. Como base de la planeación de las actividades se requiere el conocimiento de las características de este binomio. El propósito es fortalecer la relación afectiva y progresivamente habilitarlos para que de la convivencia surja de manera natural el cuidado, con el propósito de que se eviten o minimicen las fricciones y las tensiones que afectan la salud del adulto mayor y la del familiar de convivencia o potencial cuidador familiar. El conocimiento que se genera en este estudio será incorporado a los programas de teoría y práctica de enfermería; donde los estudiantes participan en la elaboración y aplicación de programas de atención a los adultos mayores en su contexto familiar.

\section{CONCLUSIONES}

Las características del adulto mayor y familiar de convivencia con relación estadísticamente significativa son la edad y el sexo. La mayoría de los adultos mayores son de mayor edad que el familiar de convivencia. La mayoría de los familiares de convivencia de los adultos mayores masculinos son mujeres. Los familiares de convivencia se caracterizan como mujeres, casadas, con bajo nivel de escolaridad y con ocupación labores del hogar. Es posible que estos sean o se conviertan en cuidadores de los adultos mayores dependientes. Este dato es relevante para considerar las acciones de promoción y prevención que le competen a enfermería, en la unidad de atención familiar, con el binomio adulto mayor-familiar de convivencia en los hogares. Las acciones estarán encaminadas a fortalecer las relaciones familiares y promover el desarrollo de habilidades en la atención a las necesidades de cuidado al adulto mayor. 


\section{REFERENCIAS BIBLIOGRÁFICAS}

1 World Health Organization. The World is fast ageing-have we noticed? The time to plan and act is now. Consultado 15 de mayo de 2009. Disponible en http://www.who. int/ageing/en

2 Secretaría de Salud. Programa de Acción: Atención al Envejecimiento. México, 2001.

3 Secretaría de Salud. Programa Nacional de Salud 20072012. Por un México sano: Construyendo alianzas para una mejor salud. 2007. Consultado el 18 de marzo de 2009. Disponible en: http://portal.salud.gob.mx/sites/descagas/pdf/pns_version_completa.pdf

4 Consejo Nacional de Población. La situación demográfica en México 2006. México. P.13

5 Instituto Nacional de Estadística, Geografía e Informática (INEGl). Encuesta Nacional sobre el Uso del Tiempo 2002. Tabulados básicos definitivos. Aguascalientes, Ags. México 2005.

6 Instituto Nacional de Salud Pública. Información científica para el cuidado de enfermería: Promoción de la salud del adulto en plenitud. Julio-septiembre 2004. Vol. 3 No. 3.

7 Hayflick L. Cómo y por qué envejecemos. Herder, España. 1999: 49-51.

8 Belsky J. Psicología del envejecimiento. Paraninfo. Thompson Learning, España. 2001

9 Nicola de, P. Geriatría. México: El Manual Moderno. 1985 :15.

10 Fericgla JM. Envejecer. Una antropología de la ancianidad. Barcelona, España: Empresa Editorial Herder, S.A. 2001.

11 Jáuregui B, Poblete E, Salgado VN. El papel de la red familiar y social en el proceso de envejecimiento en cuatro ciudades de México. En Salgado VN \& Wong R (Editoras). Envejecimiento, pobreza y saluden población urbana. Un estudio en cuatro ciudades de México. Instituto Nacional de Salud Pública. México 2006.

12 Montes V \& Hebrero M. Eventos cruciales y ciclos familiares avanzados: El efecto del envejecimiento en los hogares de México. Papeles de Población. Octubre-Diciembre No. 50. 2006. México.

13 Aguilar RM, Félix A, Martínez ML, Vega MC, Quevedo MM, Gutiérrez $\mathrm{G}$. Dependencia funcional y género del adulto mayor. Desarrollo Científico de Enfermería Vol. 15 No. 10 Noviembre-diciembre 2007: 436-439.

14 Mendoza-Parra S, Merino JM, Barriga OA. Identificación de factores de predicción del incumplimiento terapéutico en adultos mayores hipertensos de una comunidad del sur de Chile. Rev Panam Salud Pública. 2009; 25(2):10512.

15 Dorantes-Mendoza G, Ávila-Funes JA, Mejía-Arango S, Gutiérrez-Robledo LM. Factores asociados con la dependencia funcional en los adultos mayores: un análisis secundario del Estudio Nacional sobre Salud y Envejecimiento en México, 2001. Rev Panam Salud Pública. 2007; 22(1):1-11.

16 Figuererdo N, Sotolongo I, Arcias RC, Díaz G. Caracterización del adulto mayor en la comunidad. Rev Cubana Enfermer 2003; 19(3).

17 Barrios AM, Hernández C, Urbina RL. Salud mental del adulto mayor de Poza Rica de Hidalgo, Veracruz, México. Rev Enfermería Universitaria. 2009; 6(2):30-33.

18 Salcedo RA, Zarate RA, Zarza MD, Reyes V, Rubio S, Fajardo $\mathrm{G}$. La calidad de vida en los adultos mayores y su percepción en la calidad de la atención de enfermería. Rev Enfermería Universitaria. 2007; 4(3):7-12.

19 Vargas-Daza ER, García-Peña MC, Reyes-Beaman S. Satisfacción de los pacientes hipertensos de 60 años y más sobre la atención de enfermería en casa. Rev Enferm IMSS 2006; 14 (1): 3-8

20 Bover A \& Gastaldo D. La centralidad de la familia como recurso en el cuidado domiciliario: perspectivas de género y generación. Rev. bras. enferm. vol.58 no.1 Brasília Jan/Feb. 2005

21 Wiles J. Conceptualizing place in the care of older people: the contributions of geographical gerontology. 2005. Blackwell Publishing.

22. Secretaría de Salud, Reglamento de la Ley General de Salud en materia de investigación para la salud. Consultado el 18 de enero de 2007. Disponible en http://www. salud.gob.mx/unidades/cdi/nom/compi/rlgsmis.html

23 La Parra, D. La atención a la salud en el hogar: Desigualdades y tendencias. Universidad de Alicante. 2002. Pp.50

24 Salgado VN \& Wong R (Editoras). Envejeciendo en la pobreza: género, salud y calidad de vida. Instituto Nacional de Salud Pública. México 2003.

25 Ministerio de Trabajo y Asuntos Sociales. Secretaría de Estado de Servicios Sociales Familias y Discapacidad. IMSERSO. Cuidados a las personas mayores en los Hogares Españoles. El entorno familiar. Colección Estudios. Serie Dependencia. España. 2005.

\section{DIRECCIÓN PARA CORRESPONDENCIA}

Dra. Rosa Ma. Aguilar Hernández

raguilar@uat.edu.mx 